\title{
STOCHASTIC FLOWPATH ANALYSIS OF MULTIPHASE FLOW IN RANDOM POROUS MEDIA*
}

\author{
GEORGE CHRISTAKOS ${ }^{\dagger}$, DIONISSIOS T. HRISTOPULOS ${ }^{\dagger}$, AND $^{\circ}$ \\ ALEXANDER KOLOVOS ${ }^{\dagger}$
}

\begin{abstract}
Multiphase flow in random porous media is studied by means of a stochastic flowpath approach, which is built upon concepts of differential geometry. This approach replaces the partial differential equations of flow by a set of ordinary differential equations along the flowpaths. Geometrical characterization of multiphase flow involves space transformations. The stochastic flowpath approach accounts for heterogeneity and allows for random initial or boundary conditions. It does not require perturbative approximations, and it does not involve Green's functions. The differential geometric formulation involves tracking the flowpath trajectories of the different phases within the flow domain. We discuss the solution of the two-phase flow equation within a random permeability medium by numerical simulation.
\end{abstract}

Key words. random porous media, multiphase flow, differential geometry

AMS subject classifications. 35Q80, 68U20, 60G60

PII. S0036139997320548

1. Introduction. One of the most challenging problems of flow modeling in porous media is the solution of the partial differential equations (PDEs) governing multiphase flow [1], [2], [3], [4]. The main limiting factors in this problem are the heterogeneity of the flow parameters, the coupling of the flow equations for the different phases, and the nonlinearity of the constitutive relations. In addition, laboratory experiments are not sufficient for an understanding of the heterogeneities that influence flow at the field scale. Finally, field-scale simulators require averaging of the fluid properties within grid blocks of considerable sizes, thus implying an additional level of approximation [5], [6].

In light of the above considerations, both analytical and numerical treatments of the multiphase flow equations are faced with serious complications. In the case of analytical solutions, these complications include singular Green's functions, complicated boundary or initial conditions, and spatially or temporally variable inputs [7], [8]. Most numerical methods introduce approximations and simplifications, the significance of which is not always clear or well justified, they are usually very expensive to implement for two- and three-dimensional domains, and often they do not provide a deeper understanding of the physical mechanisms [9], [10], [11].

An important aspect of multiphase flow in porous media is the inherent randomness that is evident in both the geometric structure of the porous formation and the fluid flow patterns [12]. Deterministic approaches (analytical and numerical) are not sufficient in this case (although they can be used to investigate individual realizations), but stochastic methods can incorporate randomness into multiphase flow modeling. Thus, they account for the lack of a complete characterization of heterogeneity (due

*Received by the editors April 29, 1997; accepted for publication (in revised form) March 23, 1999; published electronically May 15, 2000. This research was supported by grants from the Army Research Office (grants DAAG55-98-1-0289 and DAAH04-96-1-0100), the Department of Energy (grant DE-FC09-93SR18262), and the National Institute of Environmental Health Sciences (grant P42 ES05948-02).

http://www.siam.org/journals/siap/60-5/32054.html

$\dagger$ Department of Environmental Science and Engineering, CB\#7400, University of North Carolina, Chapel Hill, NC 27599-7400 (george_christakos@unc.edu). 
to practically finite number of measurements) and provide estimates of the level of uncertainty involved in porous media characterization [3], [12], [13]. In the stochastic context, multiphase flow equations can be studied (a) by means of simulation methods that generate an ensemble of random field realizations [14], [15] and (b) in terms of low-order perturbation expansions of the corresponding statistical moments [16].

The macroscopic equations of flow in heterogeneous porous media are obtained by means of the representative elementary volume (REV) and the homogenization approach [17]. In the case of single-phase flow it can be shown rigorously that Darcy's law is obtained from the microscopic Stokes equations by means of the homogenization approach [18]. The same techniques can be used in the case of two-phase flow [19] but, due to the complexities of the two-phase system, the derivation of macroscopic laws is based on heuristic arguments. Macroscopic equations for two-phase flow in randomly heterogeneous media have also been obtained by the approach of stochastic homogenization [20].

This work is concerned with a stochastic flowpath (SFP) method for studying multiphase flow of immiscible fluids. Multiphase flow is governed by PDEs, in which the physical quantities are represented by means of random fields. The SFP method formulates the multiphase flow problem in terms of ordinary differential equations (ODEs) using concepts from differential geometry. The flow variables are determined by solving ODEs along the flowpaths, the trajectories of which are determined selfconsistently by the flow equations. The SFP method incorporates the natural heterogeneity of flow in porous media, and it allows for randomness in the initial and boundary conditions. In addition, it does not require the approximations of perturbation methods, and it does not involve Green's functions. The second-order differential flowpath equations (see (11) below) satisfied by each phase are similar to the equations of two-point seismic-ray tracing in an isotropic heterogeneous medium [21], [33]. The seismic-ray tracing problem has been solved in [21] by transforming the equations into a system of first-order ODEs in an expanded space and using a finite difference scheme. In the case of the two-phase problem, the equations are further complicated by the presence of the gravitational term and the coupling of the two phases by means of the nonlinear relative permeability relations.

While our method bears some resemblance to the streamtube [22] and streamline [23], [24] approaches for solving multiphase flow equations, it differs from them in certain important ways. These approaches, which are very efficient computationally, provide numerical solutions for the multiphase system assuming fractional flow conditions. In this case the capillary pressure is ignored, thus leading to equations for fractional flow variables which depend only on the saturation of the respective phase [25]. In contrast, the SFP approach proposed here does not neglect the capillary pressure. The streamtube and streamline simulators achieve additional improvements in computational efficiency by ignoring the gravitational force and assuming uniform initial conditions. These assumptions permit mapping analytical solutions along streamlines, using the explicit Buckley-Leverett expressions [25], [39]. Since our approach determines the flowpaths numerically, it admits nonuniform initial conditions. In addition, the streamline method calculates a single streamline corresponding to the total velocity operator and then solves the balance equations for each phase along this streamline. The SFP approach, on the other hand, focuses on calculating the flowpaths for all phases.

2. Steady-state two-phase flow. We study multiphase flow of two immiscible and incompressible fluids within a heterogeneous porous medium, the physical 
variables of which are represented by means of stochastically homogeneous random fields [26]. In addition, we consider only strong mixing random fields with finite correlation lengths. The permeability sample functions are assumed to be continuous and differentiable. Furthermore, permeability fields that are nonzero everywhere are considered. The last condition is satisfied by log-normal permeability distributions that are often used to model intrinsic permeability. Mathematical formulations based on the log-permeability of the porous medium are commonly used for single-phase flow [27] and multiphase flow [28] as well. The flow equations for the phases under steady-state conditions follow from the local Darcy's law for each phase [19] and the incompressibility condition $\nabla \cdot \boldsymbol{q}_{\alpha}(\boldsymbol{s})=0$ [3], where the subscript $\alpha$ is the phase index and $\boldsymbol{q}_{\alpha}$ is the flow vector of the phase $\alpha$. The incompressibility condition can be expressed as follows:

$$
\nabla \cdot \boldsymbol{j}_{a}(\boldsymbol{s})+\nabla \ln \frac{K_{a}(\boldsymbol{s})}{\mu_{a}} \cdot \boldsymbol{j}_{a}(\boldsymbol{s})=0
$$

where $s=\left(s_{1}, s_{2}, s_{3}\right)$ denotes a point within the flow domain. The piezometric pressure gradient $\boldsymbol{j}_{a}$ is given by

$$
\boldsymbol{j}_{a}(\boldsymbol{s})=-\nabla p_{a}(\boldsymbol{s})-\rho_{a} g \hat{\boldsymbol{x}}_{3}
$$

where $p_{a}$ is the fluid pressure, $\rho_{a}$ is the density of the phase $\alpha$, and $\hat{\boldsymbol{x}}_{3}$ is the unit vector in the vertical direction. The spatial random fields $K_{a}(s)$ represent the intrinsic permeabilities of the phases given by [29], [30]

$$
K_{a}(\boldsymbol{s})=k_{r a}(\boldsymbol{s}) K(\boldsymbol{s}),
$$

where $k_{r a}$ denote the relative permeabilities and $K(s)$ denotes the intrinsic permeability of the porous medium that is experimentally determined [29], [31]. Finally, the coefficients $\mu_{a}$ denote the dynamic viscosity of the phases. The formulation of the incompressibility equation in terms of the log-permeability random fields is standard in stochastic hydrology; it is motivated by the fact that the experimental permeability distributions are often lognormal. In reality, the subsurface is an anisotropic medium, and thus a scalar $K(\boldsymbol{s})$ is a poor assumption for deterministic multiphase models. In stochastic models, however, the anisotropy of the medium is expressed by means of the permeability correlation function [32]. Of course, this implies that the anisotropic effects become evident only after ensemble averaging. Extensions of the present model to anisotropic tensor $K(\boldsymbol{s})$ are also possible. This can be done easier if the SFP theory is formulated in terms of the phase permeabilities instead of their logarithms. Equation (1) should be solved with respect to $\boldsymbol{j}_{a}(\boldsymbol{s})$ subject to the boundary conditions and the constitutive relations (3).

The capillary pressure $p_{c}(\boldsymbol{s})$ is given by the difference between the nonwetting phase (nw) and the wetting phase $(\mathrm{w})$

$$
p_{c}(\boldsymbol{s})=p_{n w}(\boldsymbol{s})-p_{w}(\boldsymbol{s}) .
$$

The system of equations (2)-(4) is usually closed with empirical pressure-saturation (p-S) relations. These relations are based on the assumption that the capillary pressure depends only on the relative saturations of the two fluids, denoted by $S_{w}(s)$ and $S_{n w}(s)$. These functions represent the fraction of the fluid volume in each phase over the total fluid volume; thus, $S_{n w}+S_{w}=1$ everywhere. Various p-S relations are in 
use for different types of fluids and porous media. We present specific examples in section 6 . The p-S relation is generally expressed as

$$
p_{c}(\boldsymbol{s})=\Phi_{c}\left[S_{w}(\boldsymbol{s})\right]
$$

It is usually assumed, neglecting hysteresis effects, that $\Phi_{c}$ is a nonlinear, monotone function of $S_{w}$ [19]. $\Phi_{c}$ is a decreasing function of the wetting fluid saturation if $p_{c}$ is defined by (4); if $p_{c}$ is defined as $p_{c}=p_{w}-p_{n w}$, then $\Phi_{c}$ is an increasing function of the wetting fluid saturation. The relative permeabilities of the wetting and the nonwetting phase are given as functions of the wetting-fluid saturation by means of the $\mathrm{k}-\mathrm{S}$ relations

$$
k_{r, w}(s)=f_{w}\left[S_{w}(s)\right]
$$

and

$$
k_{r, n w}(s)=f_{n w}\left[S_{w}(s)\right] .
$$

While $f_{w}$ is an increasing function of the wetting-phase saturation $S_{w}$, the $f_{n w}$ is a monotonically decreasing function [29]. The p-S expression (5) and the k-S expressions (6) and (7) supplement the constitutive relations (3). The above set of equations is not unique, and it is worth noting that hysteretic constitutive relations have also been observed [31]. However, the specific choice of constitutive relations does not influence the general formulation of the SFP approach introduced in this work, at least if hysteresis is ignored. Equations (1)-(7) constitute the governing system of the two-phase flow equations; the pressure gradients are coupled via the constitutive relations that introduce a nonlinear dependence on saturation.

3. Geometric description of flowpath trajectories. The SFP analysis is based on a transformation of the multiphase flow system into a set of first-order differential equations that provide a geometric representation of the flow (Figure 1). While in the conventional analysis the pressure gradients at the nodes of the numerical grid are considered as the outputs of the multiphase flow system, in the SFP analysis the pressure gradients and the coordinates of the $\alpha$-flowpaths $(\alpha$-FP) are the outputs of the flow system. Naturally, the $\alpha$-FP trajectories depend upon the heterogeneity of the medium and the physical properties of the fluid phases.

In the SFP method the pressure gradients $\boldsymbol{j}_{a}(\boldsymbol{s})$ are analyzed into the scalar $\zeta_{a}(\boldsymbol{s})$ and the direction vector $\boldsymbol{e}_{a}(\boldsymbol{s})$ as follows:

$$
j_{a}(s)=\zeta_{a}(s) e_{a}(s)
$$

The vector $\boldsymbol{e}_{a}(\boldsymbol{s})$ determines the direction of the $\alpha$-FP trajectory and its unit Euclidean norm is equal to one, i.e., $\left|\boldsymbol{e}_{a}(\boldsymbol{s})\right|=1$. According to the definition (8) the $\alpha$-FP curves coincide with the streamlines [34] of each phase (i.e., the curves that are at every point tangent to the instantaneous phase velocity) in the case of a medium with a scalar, although possibly statistically anisotropic, saturated permeability. The flowpaths do not necessarily coincide with the pathlines [34] (i.e., the loci of the positions of the fluid particles in space as time progresses) in the case of unsteady flow.

A parametrized representation of the $\alpha$-FP $s_{a}(\lambda)$ in $R^{n}$ (where $\lambda \in U \subset R^{1}$ is a parameter that varies along the $\alpha-\mathrm{FP}$ ) is obtained in terms of the following mapping [35]:

$$
\lambda \rightarrow \boldsymbol{s}_{a}(\lambda)=\left(s_{1 a}(\lambda), \ldots, s_{n a}(\lambda)\right) \in R^{n} .
$$




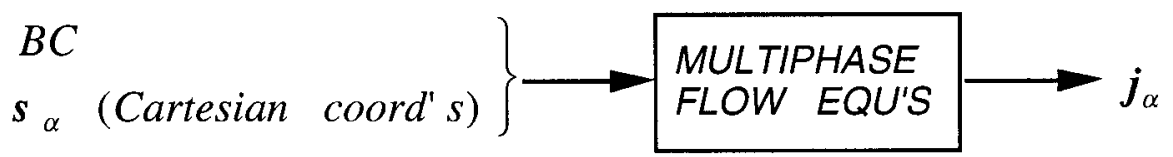

(a)

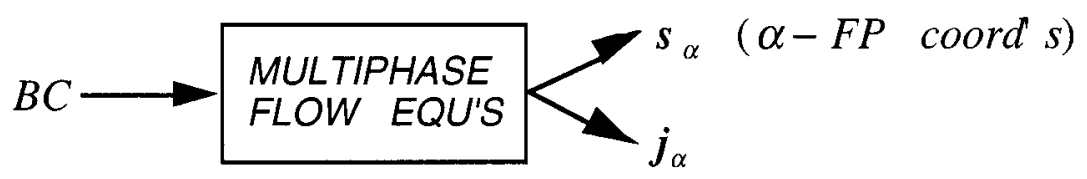

(b)

Fig. 1. (a) Conventional analysis v ( $F P=$ flowpath; $B C=$ boundary conditions $)$.

To avoid redundancy we replace $\boldsymbol{s}_{a}$ with $\boldsymbol{s}$ when it is clear which phase is denoted by $\boldsymbol{s}$, e.g., $\zeta_{a}(s)$. Equivalently, the direction of the $\alpha$-FP at each point in space is identified in terms of $n-1$ independent parameters $\gamma_{i, \alpha}(i=1, \ldots, n-1)$. An illustration is given in Figure 2 for the case of a three-dimensional Euclidean space $(n=3)$. A set of different values for the parameters $\gamma_{i, \alpha}$ leads to a family of flowpaths (Figure 3). Different choices of the parameters $\lambda$ and $\gamma_{i, \alpha}(i=1, \ldots, n-1)$ are possible. A natural choice is $\lambda=\ell$, where $\ell$ measures the total path length measured from an initial point $\mathbf{s}_{0}$ at the boundary (Figure 4). The parameters $\gamma_{i, \alpha}$ usually represent the angles of the direction vector with respect to the axes of the fixed coordinate frame, i.e., the polar and the azimuthal angles in $R^{3}$.

In a fixed Cartesian frame the direction vector $\boldsymbol{e}_{a}(\boldsymbol{s})$ is given by

$$
\boldsymbol{e}_{a}(\boldsymbol{s})=\sum_{i=1}^{n} \varepsilon_{i, a}(\boldsymbol{s}) \hat{\boldsymbol{x}}_{i},
$$

where $\hat{\boldsymbol{x}}_{i}$ are the unit vectors along the axes and $\varepsilon_{i, a}(\boldsymbol{s})$ are the direction cosines of the $\alpha$-FP

$$
\varepsilon_{i, a}(\boldsymbol{s})=\cos \theta_{i, a}(\boldsymbol{s})=d s_{i, a}(\ell) / d \ell,
$$

where $d \ell$ denotes the arc length of an infinitesimal element of the $\alpha$-FP at point $s$ along the tangential direction $\boldsymbol{e}_{a}(\boldsymbol{s})$, so that $d \ell^{2}=\sum_{i=1}^{n} d s_{i, a}^{2}$. This leads to the constraint

$$
\sum_{i=1}^{n} d s_{i, a}^{2} / d \ell^{2}=\sum_{i=1}^{n} \varepsilon_{i, a}^{2}=1 .
$$




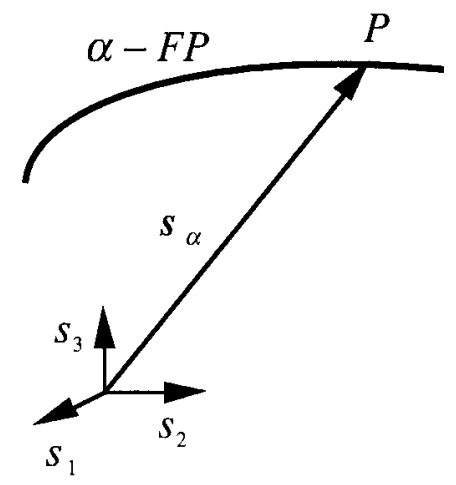

FIG. 2. An $\alpha-F P$ in $R^{3}$.

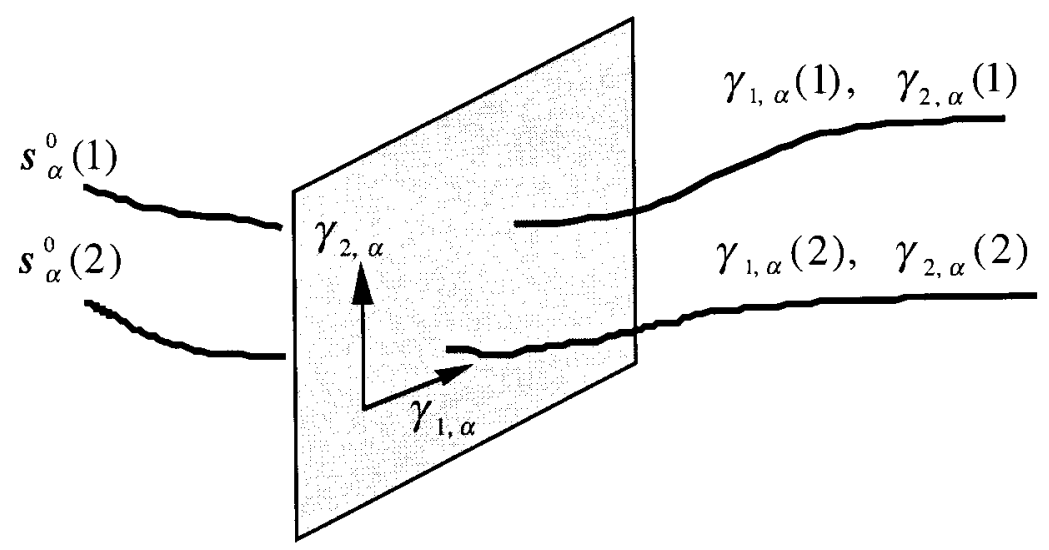

FIG. 3. A family of $\alpha-F P s$ in $R^{3}$.

Note that along an $\alpha$-FP the direction vector $\boldsymbol{e}_{a}(\boldsymbol{s})$ depends on a single parameter, i.e., the path length $\ell$. The one-dimensional derivative of a function $f(s)$ along the flowpath is given by $d f(s) / d \ell=\nabla f(s) \cdot e_{a}$. Using the geometric representations above, it can be shown that the rate of change of the pressure gradient along the $\alpha$-FP is given by (see Appendix I)

$$
\frac{d}{d \ell}\left[\zeta_{a}(s) \frac{d \boldsymbol{s}_{a}}{d \ell}\right]=\nabla \zeta_{a}(\boldsymbol{s})+\rho_{a} g \nabla \varepsilon_{3, a}(\boldsymbol{s}) .
$$

Note that according to (11) all coordinates of the flowpaths are influenced by the gravitational force, by an amount proportional to the rate of change of $\varepsilon_{3, a}$ in the specific direction.

4. Flowpath formulation of two-phase flow. In this section we develop a space transformation [26] representation of the multiphase flow along the $\alpha$-FP for the case of the initial value problem. In view of (8) above, the incompressibility 


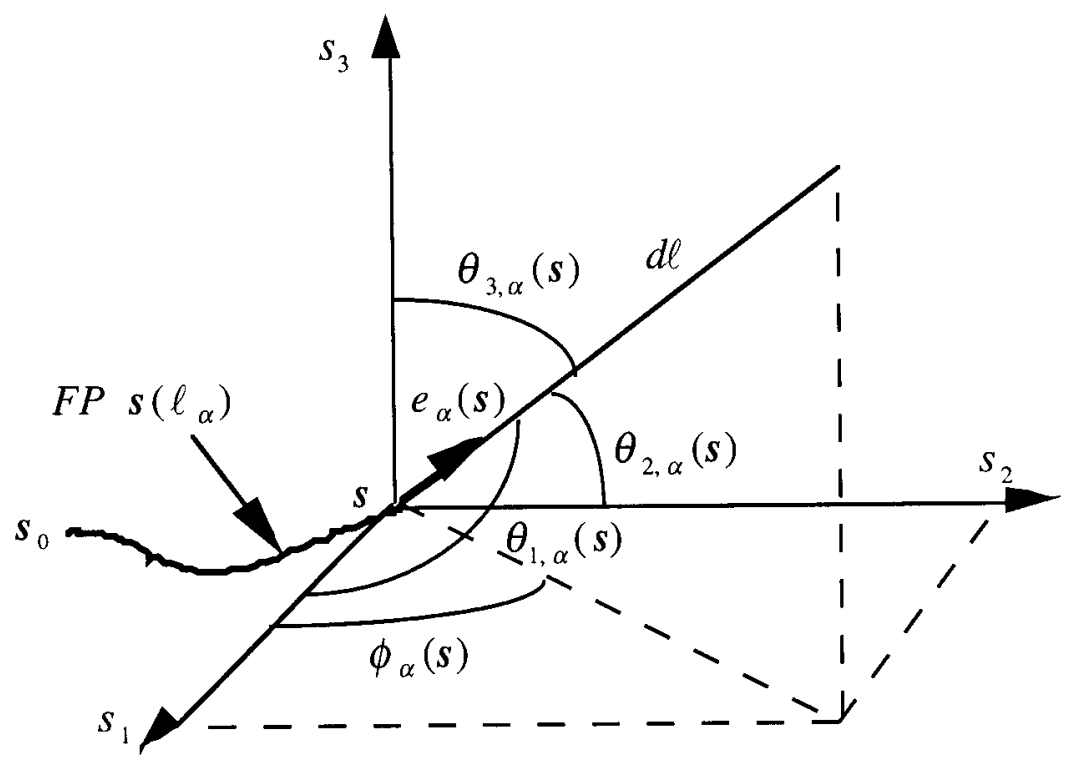

FIG. 4. A parametrized $\alpha-F P$ in $R^{3}$.

conditions, i.e., (1), can be expressed by means of stochastic ODEs as follows:

$$
\frac{d \zeta_{a}(s)}{d \ell}+\left\{\nabla \cdot e_{a}(s)+\frac{d}{d \ell}\left[\ln \frac{K_{a}(s)}{\mu_{a}}\right]\right\} \zeta_{a}(s)=0 .
$$

Note that neighboring flowpaths of each phase are coupled via the term that involves the divergence of the direction vector $\boldsymbol{e}_{a}$.

The flux along the flowpath is defined by $\boldsymbol{q}_{a}(\boldsymbol{s})=\frac{K_{a}(\boldsymbol{s})}{\mu_{a}} \zeta_{a}(\boldsymbol{s}) \boldsymbol{e}_{a}(\boldsymbol{s})$, and the scalar flux $\left\|\boldsymbol{q}_{a}(\boldsymbol{s})\right\|$ is denoted by $q_{a}(\boldsymbol{s})$. For the initial value problem the general solution of (12) along the $\alpha$-FP is then

$$
q_{a}(\boldsymbol{s})=q_{a}\left(\boldsymbol{s}_{0}\right) \exp \left[-\eta_{a}(\boldsymbol{s})\right]
$$

where $\eta_{a}(\boldsymbol{s})$ is the space transformation of the divergence of $\boldsymbol{e}_{a}$ along the $\alpha$-FP, given by

$$
\eta_{a}(\boldsymbol{s})=\eta_{a}(\ell)=\int_{\boldsymbol{s}_{a}(0)}^{\boldsymbol{s}_{a}(\ell)} \nabla \cdot \boldsymbol{e}_{a}\left(\boldsymbol{s}^{\prime}\right) d \ell^{\prime} .
$$

In the special case that the $\alpha$-FP is a straight line in a two-dimensional domain, (14) is the standard Radon transform [36]. More generally, if the $\alpha$-FP is an arbitrary curve, (14) defines a nonlinear extension of the Radon transform [37]. An equivalent expression for $\eta_{a}(\boldsymbol{s})$ is as follows:

$$
\eta_{a}(s)=\sum_{i=1}^{n} \int_{s_{i, \alpha}(0)}^{s_{i, \alpha}(\ell)} \frac{1}{\varepsilon_{i, a}\left(s \mid u_{i}\right)} \frac{\partial \varepsilon_{i, a}\left(s \mid u_{i}\right)}{\partial u_{i}} d u_{i},
$$

where the $\left(s \mid u_{i}\right)$ denotes the vector $\left(s_{1}, \ldots, s_{i-1}, u_{i}, s_{i+1}, \ldots, s_{n}\right)$. Equations (13)-(15) provide closed-form expressions for $\zeta_{\alpha}$ in terms of the initial conditions $\zeta_{\alpha}\left(s_{0}\right)$, the 
permeability field $K_{\alpha}$, and the space transformation $\eta_{\alpha}$. However, explicit solutions for the $\zeta_{\alpha}$ require the flowpath equations.

The incompressibility equations (12) are complemented by a set of equations that determine the flowpaths. In particular, (11) leads to the following $2 \times n$ first-order, nonlinear differential equations:

$$
\frac{d}{d \ell}\left[\zeta_{a}(\boldsymbol{s}) \cos \theta_{i, a}(\boldsymbol{s})\right]=\frac{\partial \zeta_{a}(\boldsymbol{s})}{\partial s_{i}}+\rho_{a} g \frac{\partial \cos \theta_{3, a}(\boldsymbol{s})}{\partial s_{i}}, \quad i=1, \ldots, n .
$$

Note that for $n>1$ the variables $\theta_{i, a}$ are not independent, since they are constrained by $\sum_{i=1}^{n} \theta_{i, a}^{2}=1$. Accordingly, only $2 \times(n-1)$ of the equations (16) are independent. Equations (12) and (16) form the SFP system, which is closed by the constitutive relationships (3)-(7) and subject to the initial and boundary flow conditions. Since the $\mathrm{p}-\mathrm{S}$ relation involves the pressure difference of the two phases, additional relations are required between the phase pressures, the pressure gradients, and the flowpaths; these are obtained by integrating (I.2) as follows:

$$
p_{a}(\boldsymbol{s})=p_{a}\left(\boldsymbol{s}_{0}\right)-\int_{\boldsymbol{s}_{\alpha}(0)}^{\boldsymbol{s}_{\alpha}(\ell)} d \ell^{\prime}\left[\zeta_{a}\left(\boldsymbol{s}^{\prime}\right)+\rho_{a} g \varepsilon_{3, a}\left(\boldsymbol{s}^{\prime}\right)\right] .
$$

Either deterministic or random flow conditions can be considered with the SFP method; the type of boundary conditions is expected to affect the flow solutions as shown in [38] in the case of single-phase flow. Finally, note that another useful formulation of the multiphase flow equations along the $\alpha$-FP is obtained in terms of the Jacobian of the transformation from the Cartesian to the flowpath coordinates [37].

5. Two-phase flow in a layered heterogeneous medium. In this section we consider flow in a porous medium with uniaxial heterogeneity in the vertical direction. This scenario applies, e.g., to layered media that exhibit homogeneous intrinsic permeabilities within the layers with variations only along $s_{3}$. In this case, (14), which determines the variation of $\boldsymbol{e}_{\alpha}$ between an initial point $\boldsymbol{s}_{0}$ and an endpoint $\boldsymbol{s}_{f}$ of an $\alpha$-FP, leads to the algebraic expression

$$
\eta_{a}\left(\boldsymbol{s}_{f}\right)=\ln \left[\frac{\cos \theta_{3, a}\left(\boldsymbol{s}_{f}\right)}{\cos \theta_{3, a}\left(\boldsymbol{s}_{0}\right)}\right]
$$

Equation (13) governs the variation of the flux along the flowpath. In view of (13) and (18) the pressure gradient at the endpoint is determined by

$$
\frac{\zeta_{a}\left(\boldsymbol{s}_{f}\right)}{\zeta_{a}\left(\boldsymbol{s}_{0}\right)}=\frac{K_{a}\left(\boldsymbol{s}_{0}\right)}{K_{a}\left(\boldsymbol{s}_{f}\right)} \frac{\cos \theta_{3, a}\left(\boldsymbol{s}_{0}\right)}{\cos \theta_{3, a}\left(\boldsymbol{s}_{f}\right)}
$$

The flowpath equations (16) give $\zeta_{a}(\boldsymbol{s}) \cos \theta_{i, a}(\boldsymbol{s})=c_{i}$, for $i=1,2$. It then follows that the magnitude of the pressure gradient is $\zeta_{a}(s)=j_{\perp, a} / \sin \theta_{3, a}(s)$, where $j_{\perp, a}=$ $\sqrt{c_{1}^{2}+c_{2}^{2}}$. In view of this relationship, (19) leads to

$$
\frac{\tan \theta_{3, a}\left(\boldsymbol{s}_{f}\right)}{K_{a}\left(\boldsymbol{s}_{f}\right)}=\frac{\tan \theta_{3, a}\left(\boldsymbol{s}_{0}\right)}{K_{a}\left(\boldsymbol{s}_{0}\right)} .
$$

Therefore, the flowpaths are determined by the initial conditions and the intrinsic permeabilities. The pressures along the flowpaths are given by the integral in (17). 
Thus, we obtain the following nonlinear system of integral equations for the phase pressures:

$$
p_{a}\left(\boldsymbol{s}_{f}\right)=p_{a}\left(\boldsymbol{s}_{0}\right)-\rho_{a} g \hat{\boldsymbol{x}}_{3} \cdot\left(\boldsymbol{s}_{f}-\boldsymbol{s}_{0}\right)-\int_{\boldsymbol{s}_{0}}^{\boldsymbol{s}_{f}} d s_{3}^{\prime} \frac{\mu_{a} q_{3, a}\left(\boldsymbol{s}_{0}\right)}{K_{a}\left(\boldsymbol{s}^{\prime}\right) \cos \theta_{3, a}^{2}\left(\boldsymbol{s}^{\prime}\right)} .
$$

The intrinsic permeabilities depend implicitly on the saturation, and thus the integral cannot be directly evaluated. In fact, (20) and (21) along with the appropriate k-S relationships and the p-S relation form a system of $3 \alpha+1$ equations containing an equal number of unknowns, e.g., $p_{a}, \theta_{3, a}, K_{a}$, and $S_{e}$. Note that the differential equations (12) and (16) have been replaced with the algebraic equations (19) and (20). Finally, in the case of saturated, single-phase flow, (20) yields the well-known law of streamline refraction [34].

6. Waterflooding of a heterogeneous oil reservoir. In the following we consider injection of water (wetting phase) in a two-dimensional, heterogeneous oil reservoir. We consider boundary conditions for the saturation coefficient and the pressure gradients (magnitude $\zeta_{a}\left(s_{0}\right)$ and incidence angles $\theta_{1, a}\left(s_{0}\right)$ ) of the $\alpha$-FPs given along a boundary line of a square grid. The gravitational term is ignored in two dimensions. The two-phase flow satisfies (12) and (16). The intrinsic log-permeability, $\ln K_{s}$, is modeled using stochastically homogeneous random fields [26]. We use the Brooks-Corey relations for the p-S and the k-S curves. These are expressed in terms of the reduced water saturation $S_{e}(s)$, defined by

$$
S_{e}(s)=\frac{S_{w}(s)-S_{w, r e s}}{S_{w, s a t}-S_{w, r e s}}
$$

where $S_{w, r e s}$ denotes the residual and $S_{w, s a t}$ the maximum saturation of the wetting phase. The reduced saturation takes values in $[0,1]$. Correspondingly, the reduced oil saturation is given by $1-S_{e}(\boldsymbol{s})$. Explicit expressions for the relative permeabilities of the oil-water system are given by the following k-S relations [34]:

$$
k_{r w}(s)=k_{r w}^{0}\left[S_{e}(s)\right]^{(2+3 \lambda) / \lambda}
$$

and

$$
k_{r o}(s)=k_{r o}^{0}\left[1-S_{e}^{(2+\lambda) / \lambda}(s)\right]\left[1-S_{e}(s)\right]^{2},
$$

where $k_{r o}^{0}, k_{r w}^{0}$, and $\lambda$ are empirical parameters that characterize the medium. The relation between the capillary pressure and the water saturation is modeled by means of

$$
S_{e}(\boldsymbol{s})= \begin{cases}{\left[p_{c}(\boldsymbol{s}) / p_{e}\right]^{-\lambda},} & p_{c}>p_{e} \\ 1, & p_{c} \leq p_{e}\end{cases}
$$

where $p_{e}$ denotes the capillary pressure at maximum water saturation. Plots of the $\mathrm{k}-\mathrm{S}$ relations are shown in Figure 5 for three different values of the parameter $\lambda$. For a fixed $\lambda$, the $k_{r w}$ is an increasing function of $S_{e}$ while the $k_{r o}$ is decreasing. For a fixed $S_{e}$, the $k_{r w}$ is an increasing function of $\lambda$ while the $k_{r o}$ is decreasing. The p-S behavior is shown in Figure 6: The p-S curve is a monotonically decreasing function of $S_{e}$. In addition, for a given $S_{e}$ the capillary pressure decreases with increasing $\lambda$. In the following, $k_{r o}^{0}=0.9, k_{r w}^{0}=0.5, S_{w, r e s}=0.1, S_{w, s a t}=0.9$. The dynamic viscosity 


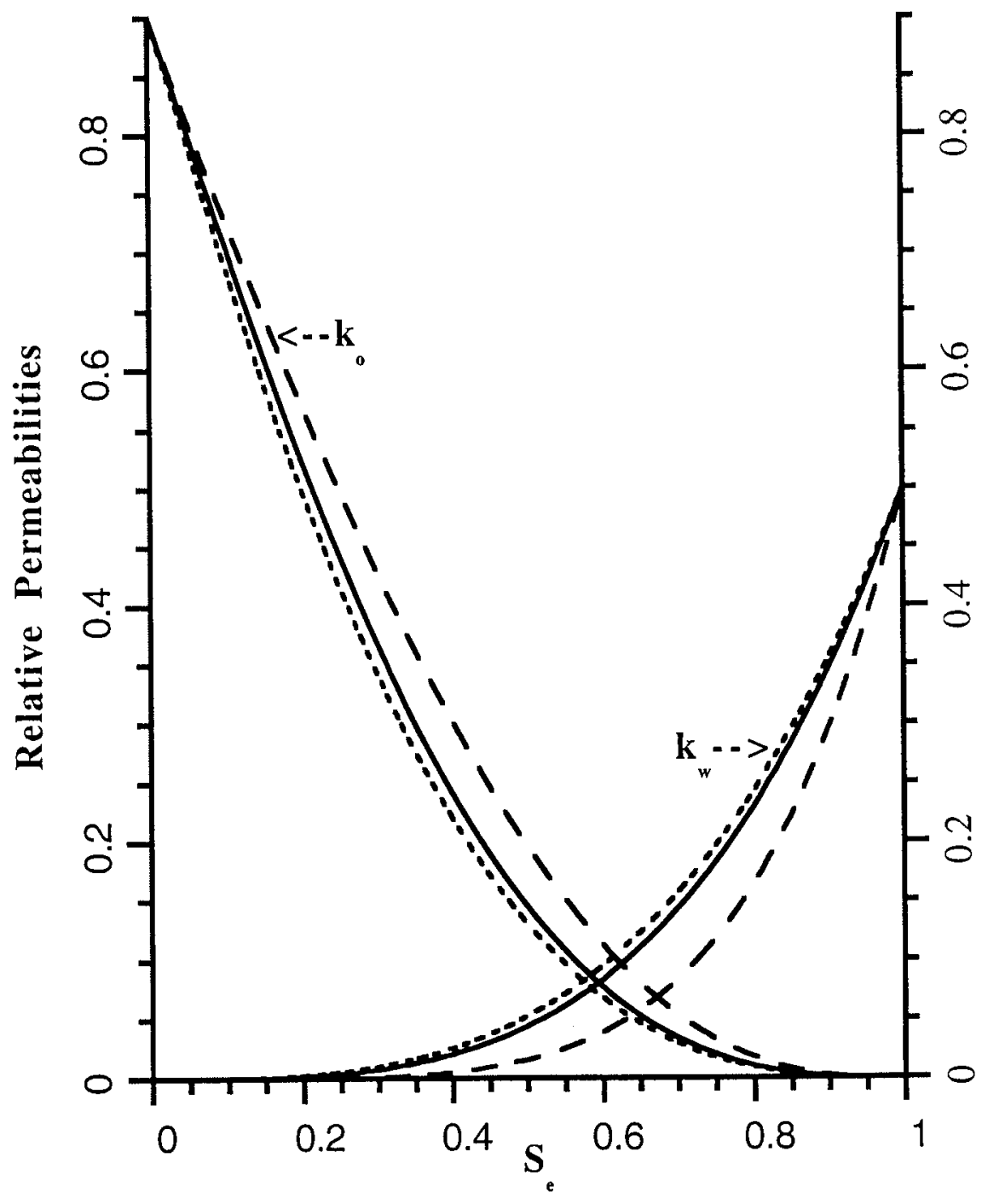

Fig. 5. Plots of the Brooks-Corey $k-S$ curves for $\lambda=1$ (dashes), $\lambda=4$ (solid line), and $\lambda=8$ (dots).

of the water is $\mu_{w}=5 \times 10^{-3}$ poise, and the viscosity of oil $\mu_{o}=1 \times 10^{-1}$ poise. The pressure is expressed in units of $p_{e}$.

The complete system of two-phase flow equations involves two incompressibility conditions (12), two independent flowpath equations (16), four constitutive relationships (4) and (23)-(25), and two pressure integrals (17). Thus, the total number of equations is 10 , matching the number of unknowns: $\zeta_{a}, \theta_{1, a}, p_{a}, k_{r, a}(\alpha=w$ and o), $S_{e}$, and $p_{c}$. The above two-phase flow system is investigated by means of numerical simulations. 


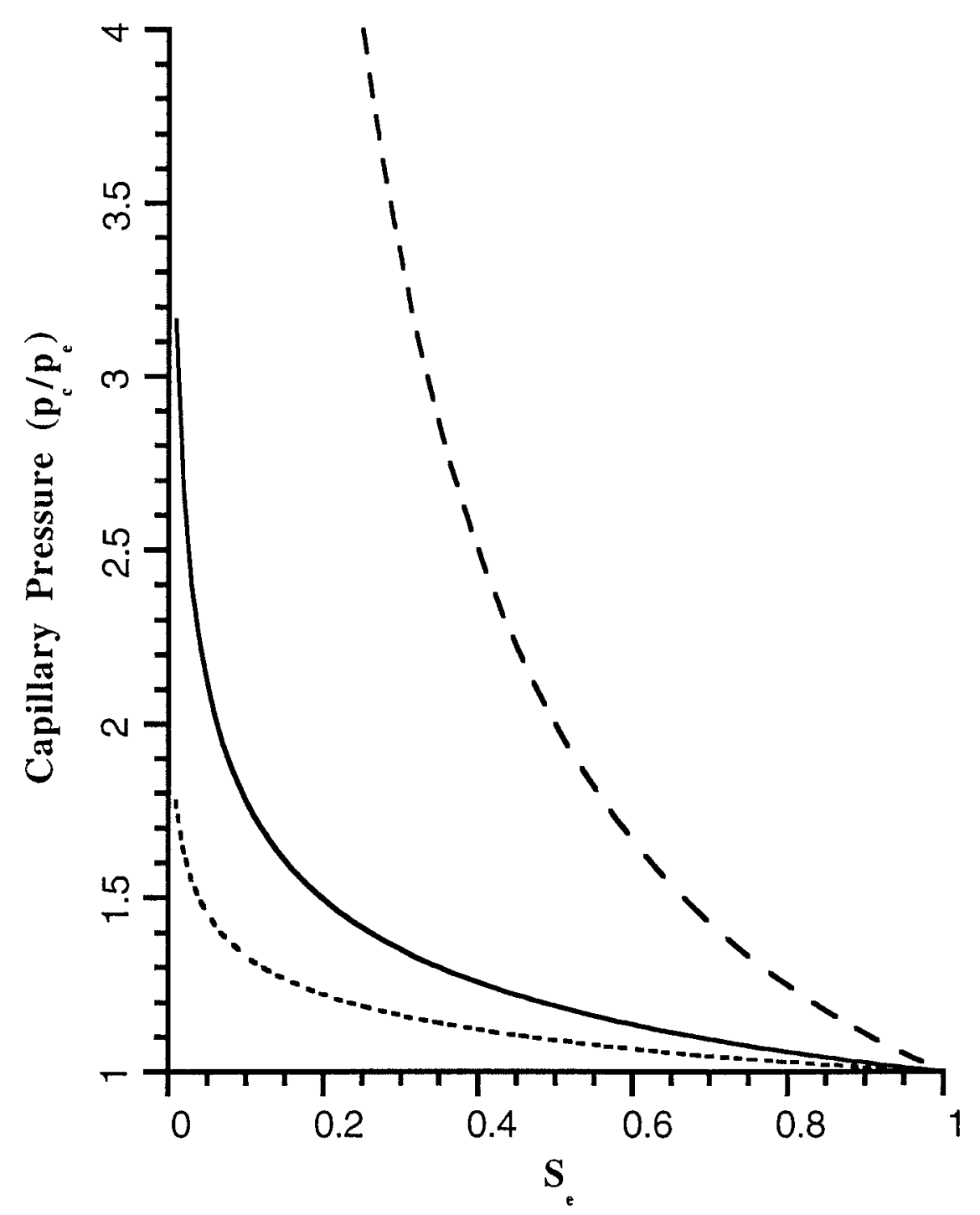

FiG. 6. Plots of the $p-S$ curves for $\lambda=1$ (dashes), $\lambda=4$ (solid line), and $\lambda=8$ (dots).

7. Numerical solutions. The variables of the flowpath system are the pressure gradients and the direction of the flowpaths that correspond to the two phases. The flowpath equations represent the evolution of each phase along different flowpaths; however, the relative permeabilities of the phases at every point depend on the capillary pressure, and thus on the pressure of the other phase at this point. This coupling of the flowpaths prevents a straightforward solution of the ODEs by, e.g., RungeKutta or other first-order nonlinear ODE solvers. Instead, we approximate the ODEs by means of difference equations that we solve using the Newton-Raphson method [40]. The saturation is also an unknown variable to be determined from the solution 
of the flowpath system.

We propose to solve the steady-state flow equations using an iterative numerical approach that assumes a profile $S_{e, 0}(\boldsymbol{s})$ as an initial guess for the saturation coefficient. This determines the initial capillary pressure and the intrinsic permeabilities, and a reduced system of four first-order nonlinear differential equations is obtained - which includes the incompressibility conditions (12) and the independent flowpath equations (16). Solution of these equations yields the oil and water pressure gradients and the local direction of the flowpaths throughout the entire domain. The oil and water pressures along the flowpaths are evaluated by the discretized integral of the pressure gradients (17), and an updated capillary pressure profile is derived by the difference between the oil and the water pressure; based on this an updated saturation profile is obtained via (25). The reduced system is solved iteratively until a stable saturation profile is obtained. The steady-state solution should depend on the conditions at the injection boundary. It should be independent of the initial guess of the saturation profile. However, the convergence of the Newton-Raphson method in general depends on the initial guess. We have experimented with different forms of the initial profile (guess) and found that there is a layer near the boundary within which the saturation rises from the boundary value toward one (fully water-saturated medium). Outside this layer, there are regions of trapped oil with lower water saturation. We have tried different functional dependences for the initial saturation: a constant profile, linear increase, and exponential increase toward a maximum saturation. We found that the method converges fastest when the latter profile is used as an initial guess. In addition, the saturation toward which the solution converges is independent of the maximum saturation of the initial profile, at least within the range of values that we investigated. This method accomplishes a decoupling of the flow equations that allows their solution independently along the flowpaths.

We use a numerical grid with $101 \times 101$ nodes, and the distance between nodes is taken equal to one meter. The position on the grid is denoted here by $s=(x, y)$. The saturated permeability $K(\boldsymbol{s})$ is assumed to be log-normally distributed with arithmetic mean $\bar{K}=10^{-9} \mathrm{~m}^{2}$ and coefficient of variation $(\mathrm{COV}) \mu_{K}=\sigma_{K} / \bar{K}_{s}=0.2$. A Gaussian covariance model is assumed for the log-permeability fluctuations $f(x, y)$, i.e.,

$$
c_{f}(s)=\sigma_{f}^{2} \exp \left[-\left(\frac{x^{2}}{b_{x}^{2}}+\frac{y^{2}}{b_{y}^{2}}\right)\right]
$$

We investigate two cases - an isotropic correlation model with $b_{x}=b_{y}=20 \mathrm{~m}$ and an anisotropic model with $b_{x}=20 \mathrm{~m}$ and $b_{y}=40 \mathrm{~m}$. The initial conditions include the directions of the pressure gradients, $\theta_{a}\left(\boldsymbol{s}_{0}\right)=v_{a, s_{0}}$, where $v_{a, s_{0}}$ is a zero-mean, uniformly distributed random noise, the magnitude of the pressure gradients $\zeta_{n w}\left(s_{0}\right)=0.01$ and $\zeta_{w}\left(s_{0}\right)=0.03$, and the boundary saturation $S_{e}\left(s_{0}\right)=0.745$. The parameter $\lambda$ in the Brooks-Corey relations is assumed to have the value $\lambda=0.45$ in both cases. The initial guess for the saturation profile increases exponentially from the boundary and approaches a maximum level of 0.92 .

We discretize the equations using finite differences along the flowpaths. The length of the flowpaths is increased by the finite step $\delta \ell_{\alpha}$. Here we consider $\delta \ell_{o}=$ $\delta \ell_{w}=\delta \ell$. The size of the step is bounded below by the grid resolution and above by the correlation length, i.e., $\varepsilon \leq \delta \ell \ll \xi$, where $\varepsilon$ is the lattice step and $\xi$ is the correlation length of the permeability fluctuations. We use forward finite differences for the derivatives and the trapezoidal rule for function evaluations. The length 
increments in the orthogonal directions of the grid are given by

$$
\begin{aligned}
& \delta x_{a, m} \equiv x_{a, m+1}-x_{a, m}=\frac{\delta \ell}{2}\left(\cos \theta_{a, m+1}+\cos \theta_{a, m}\right), \\
& \delta y_{a, m} \equiv y_{a, m+1}-y_{a, m}=\frac{\delta \ell}{2}\left(\sin \theta_{a, m+1}+\sin \theta_{a, m}\right), \ldots, N_{f, i},
\end{aligned}
$$

and the index $m$ denotes the $m$ th point on the flowpath. The integer $N_{f, i}$ denotes the total number of increments in the $i$ th flowpath. In view of $(25),(12)$ is discretized as follows, where $F_{m}=\ln k_{m}(s)$ :

$$
\begin{aligned}
\left(\zeta_{a, m+1}\right. & \left.-\zeta_{a, m}\right)+\left[\left(F_{m+1}-F_{m}\right)+\left(\ln k_{r, a, m+1}-\ln k_{r, a, m}\right)\right. \\
- & \left.\left(\theta_{a, m+1}-\theta_{a, m}\right) w\left(\theta_{a, m}, \theta_{a, m+1}\right)\right]\left(\frac{\zeta_{a, m}+\zeta_{a, m+1}}{2}\right)=0 .
\end{aligned}
$$

The function $w\left(\theta_{a, m}, \theta_{a, m+1}\right)$ depends only on the local direction of the flowpaths and is given by

$$
w\left(\theta_{\alpha, m}, \theta_{\alpha, m+1}\right)=\frac{\sin \theta_{\alpha, m+1}+\sin \theta_{\alpha, m}}{\cos \theta_{\alpha, m+1}+\cos \theta_{\alpha, m}}-\frac{\cos \theta_{\alpha, m+1}+\cos \theta_{\alpha, m}}{\sin \theta_{\alpha, m+1}+\sin \theta_{\alpha, m}} .
$$

Equation (16) leads to the following difference equations:

$$
\zeta_{a, m+1} \cos \theta_{a, m+1}-\zeta_{a, m} \cos \theta_{a, m}=\frac{2\left(\zeta_{a, m+1}-\zeta_{a, m}\right)}{\cos \theta_{a, m+1}+\cos \theta_{a, m}}
$$

and

$$
\zeta_{a, m+1} \sin \theta_{a, m+1}-\zeta_{a, m} \sin \theta_{a, m}=\frac{2\left(\zeta_{a, m+1}-\zeta_{a, m}\right)}{\sin \theta_{a, m+1}+\sin \theta_{a, m}} .
$$

Note that when $\theta_{a} \cong 0$ the denominator of the right-hand side term of (31) tends to zero; this indicates that the flowpath is almost parallel to the $x$-axis, so that the flowpath variables do not vary along the $y$-axis. Similarly, the denominator of (30) tends to zero around $\theta_{a} \cong \pi / 2$, i.e., when the flowpath is almost parallel to the $y$-axis. Hence, when $\bmod \left(\theta_{a}, \pi / 2\right) \leq \pi / 4$ (where "mod" denotes the modulo function), (30) is automatically selected, while (31) is used when $\bmod \left(\theta_{a}, \pi / 2\right)>\pi / 4$.

Equations (28)-(31) involve the unknown variables $\theta_{\alpha, m+1}$ and $\zeta_{\alpha, m+1}$. The $\theta_{\alpha, m}$ and $\zeta_{\alpha, m}$ values are obtained from the previous step or the initial conditions at the injection boundary. The log-permeability field is known on the grid. Since the traces of the flowpaths do not pass from the grid nodes, estimates of the log-permeability at off-grid points are required. In general, such estimates can be derived by a linear kriging estimator [26]. However, if the correlation length significantly exceeds the length increment, i.e., $\delta \ell \ll \xi$, the log-permeability of the grid is accurately estimated by the arithmetic mean of the permeability at the four nearest grid points.

We denote by $N_{m}^{*}$ the number of updated nodes at the $m$ th iteration. To estimate the relaxation of the saturation profile we use the relative change in the saturation coefficient $\Delta S_{e, m}$ defined by means of

$$
\Delta S_{e, m}=\frac{1}{N_{m}^{*}} \sum_{i=1}^{N_{m}^{*}} \frac{\left|S_{e, m}\left(\boldsymbol{s}_{i}\right)-S_{e, m-1}\left(\boldsymbol{s}_{i}\right)\right|}{S_{e, m-1}\left(\boldsymbol{s}_{i}\right)} .
$$




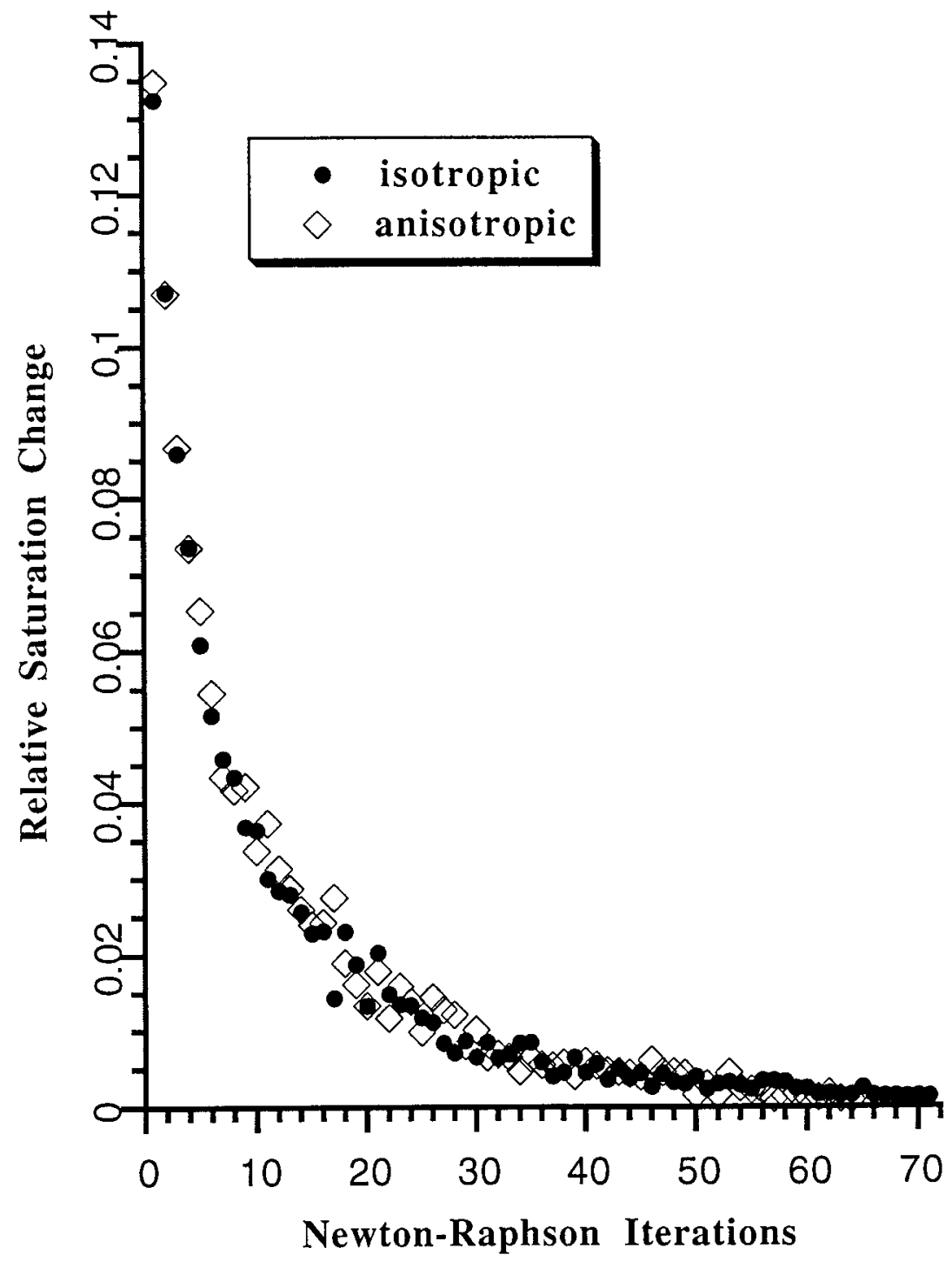

FIG. 7. Plot of the mean water saturation in the domain midpoint vs. the number of NewtonRaphson interations.

A plot of $\Delta S_{e, m}$ vs. the Newton-Raphson iteration number is shown in Figure 7. At the end of the Newton-Raphson cycle the relative saturation change is below $5 \%$ for $99.9 \%$ of the updated points, in both the isotropic and anisotropic cases. In Figure 8 we plot the mean saturation of the domain vs. the number of iterations. The percentage of nodes where the saturation is updated is plotted vs. the number of iterations in Figure 9. It is seen that this quantity essentially relaxes to a value about 0.16 after 20 iterations, although significant fluctuations persist. The resulting isopressure 


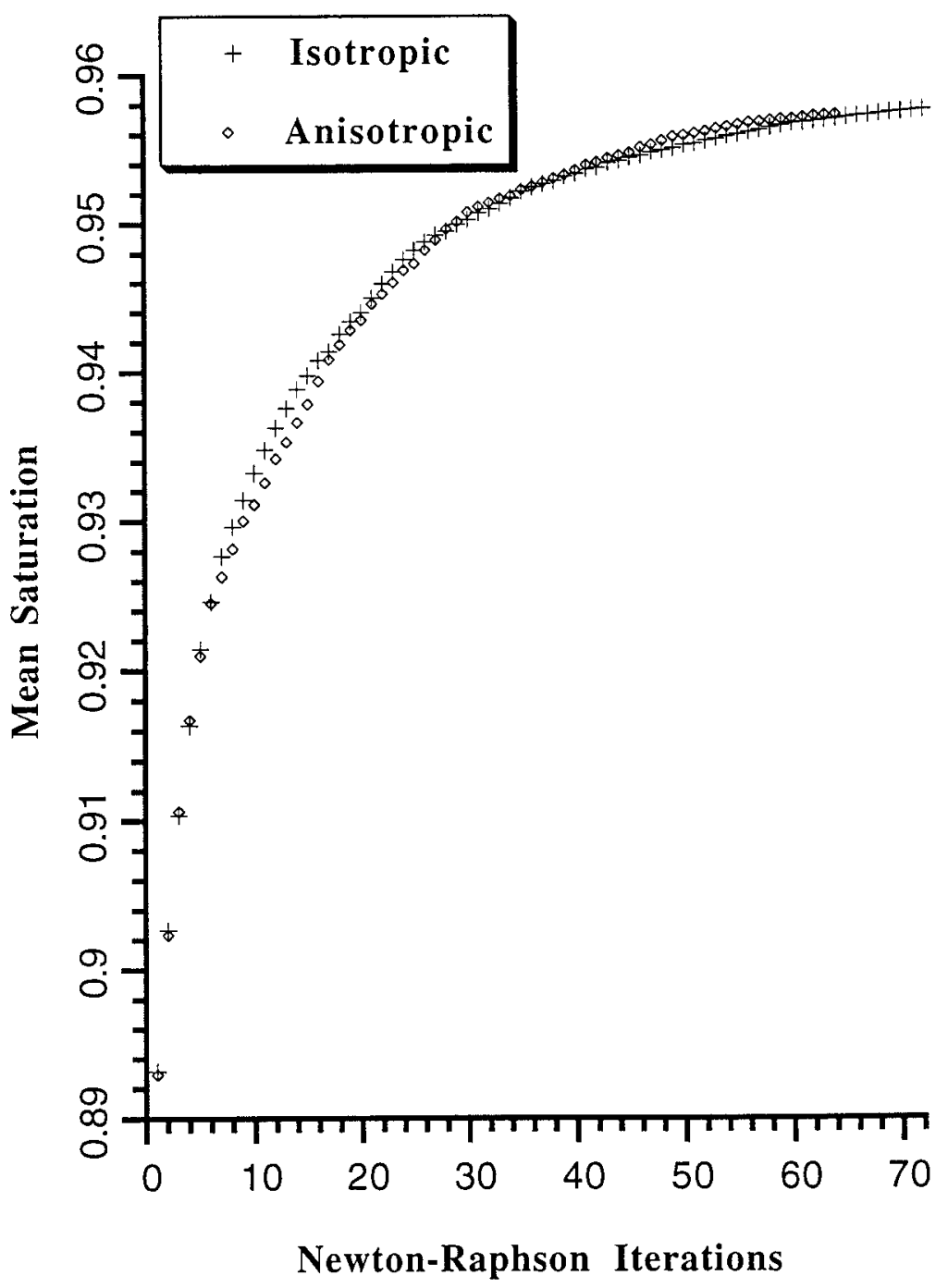

FIG. 8. Plot of the relative change in the saturation profile between consecutive Newton-Raphson iterations.

contours for the isotropic cases are shown in Figures 10 (water) and 11 (oil). The constant saturation contours are shown in Figure 12. The code generates the pressures at the nodes visited by the flowpaths, e.g., 7792 nodes for the water phase and 2622 nodes for the oil phase in the isotropic case. These numbers are similar in the anisotropic case. We use a kriging estimator to obtain the pressures at the nodes that are not visited by the flowpaths. These values are used for generating the contours in Figures 10-12. The water-phase pressure contours closely resemble single-phase flow. This is expected since, after water injection, most of the reservoir is flooded with water except for areas of trapped oil. In the saturation contour plot (Figure 12) 


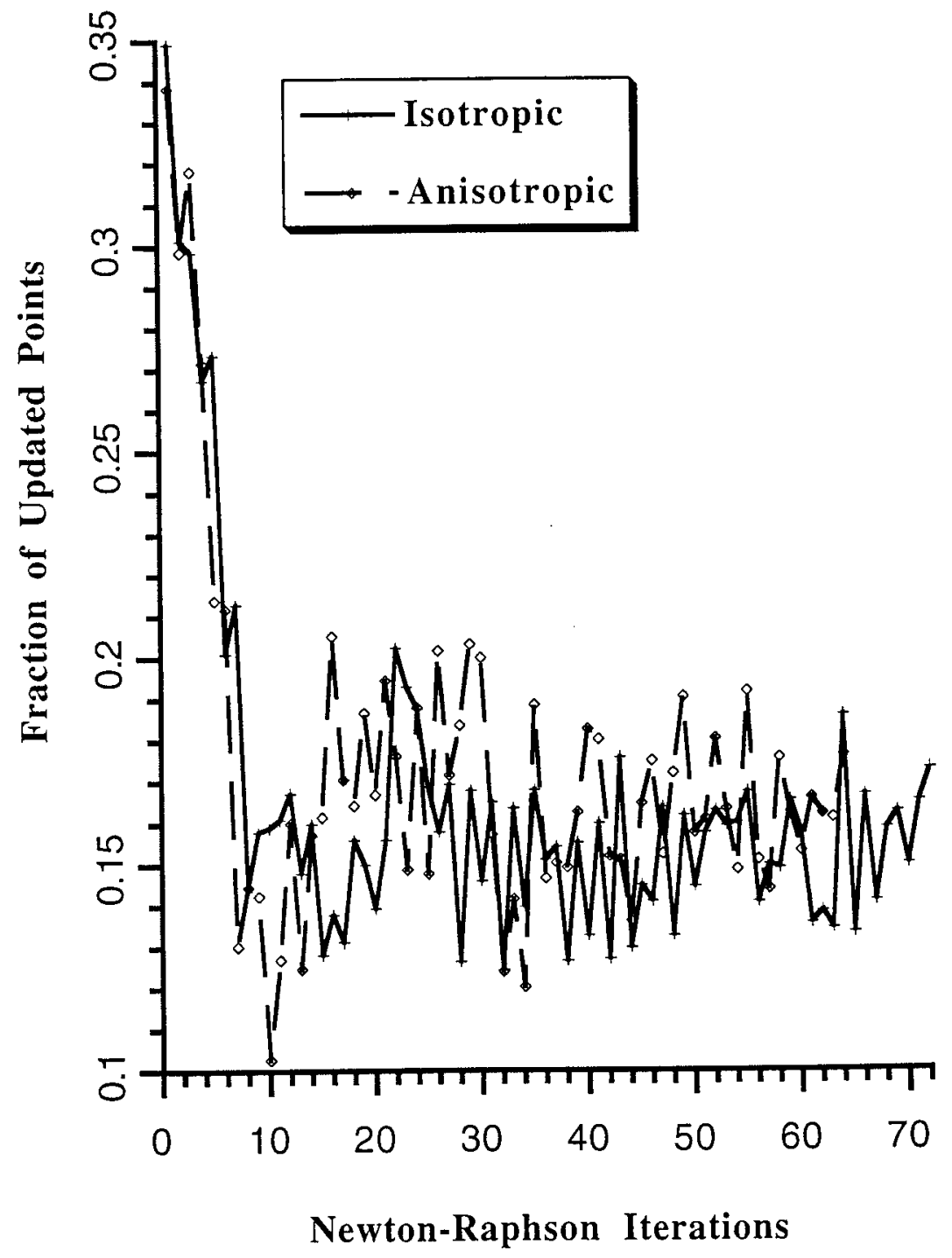

FIG. 9. Plot of the fraction of the grid points at which the saturation updated vs. the number of Newton-Raphson iterations.

there is a narrow layer near the boundary within which the water saturation orderly increases toward a maximum close to 0.99. In the anisotropic case the water phase pressure pattern (not shown) does not exhibit any significant differences from the isotropic case. The same is true of the saturation contours (shown in Figure 14). This indicates that the anisotropy of the intrinsic permeability is not a significant factor in determining the residual distribution of oil. On the other hand, the oil isopressure contours for the anisotropic intrinsic permeability field, shown in Figure 13, exhibit 
Isopressure Contours; Water Phase; Isotropic Intrinsic Permeability

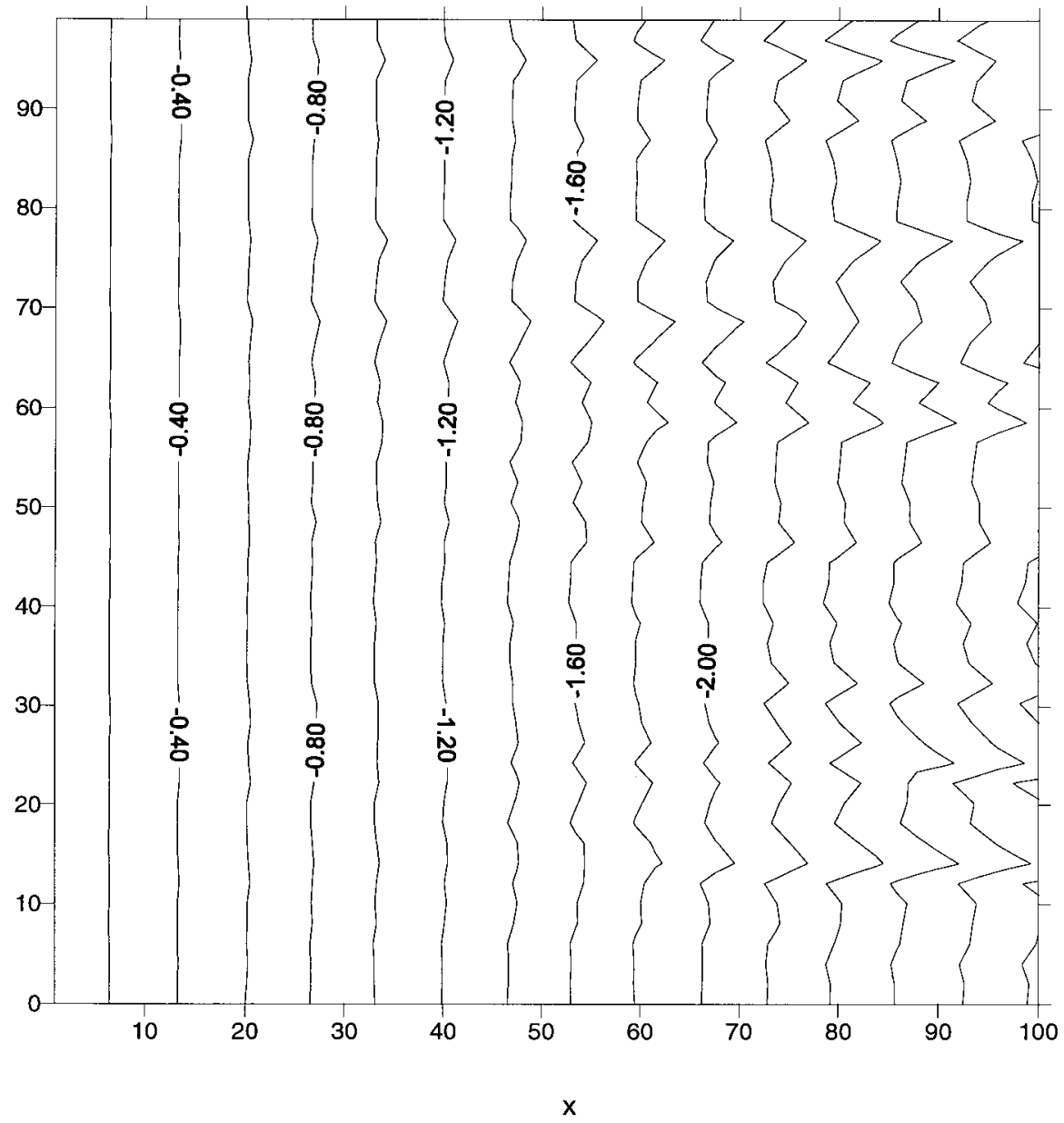

FIG. 10. Wetting phase isopressure contours for the isotropic intrinsic permeability field. The pressure is given in units of the entry pressure $p_{e}$.

significant changes with respect to the isotropic case (this behavior, however, may be an artifact, due to the relatively low number of nodes where the oil pressure is calculated).

8. Transient effects in multiphase flow. In the transient case, the dynamic flow equations for multiphase flow are given by

$$
\frac{\phi}{K_{a}(\boldsymbol{s}, t)} \frac{\partial S_{a}(\boldsymbol{s}, t)}{\partial t}+\nabla \cdot \boldsymbol{j}_{a}(\boldsymbol{s}, t)+\left[\nabla \ln \frac{K_{a}(\boldsymbol{s}, t)}{\mu_{a}}\right] \cdot \boldsymbol{j}_{a}(\boldsymbol{s}, t)=0,
$$

where $\phi$ denotes the porosity of the medium and $S_{\alpha}$ the saturation of each phase. In view of (31), (12) is replaced by

$$
\frac{\phi}{K_{a}(\boldsymbol{s}, t)} \frac{\partial S_{a}(\boldsymbol{s}, t)}{\partial t}+\frac{d \zeta_{a}(\boldsymbol{s}, t)}{d \ell}+\left\{\nabla \cdot \boldsymbol{e}_{a}(\boldsymbol{s}, t)+\frac{d}{d \ell}\left[\ln \frac{K_{a}(\boldsymbol{s}, t)}{\mu_{a}}\right]\right\} \zeta_{a}=0
$$




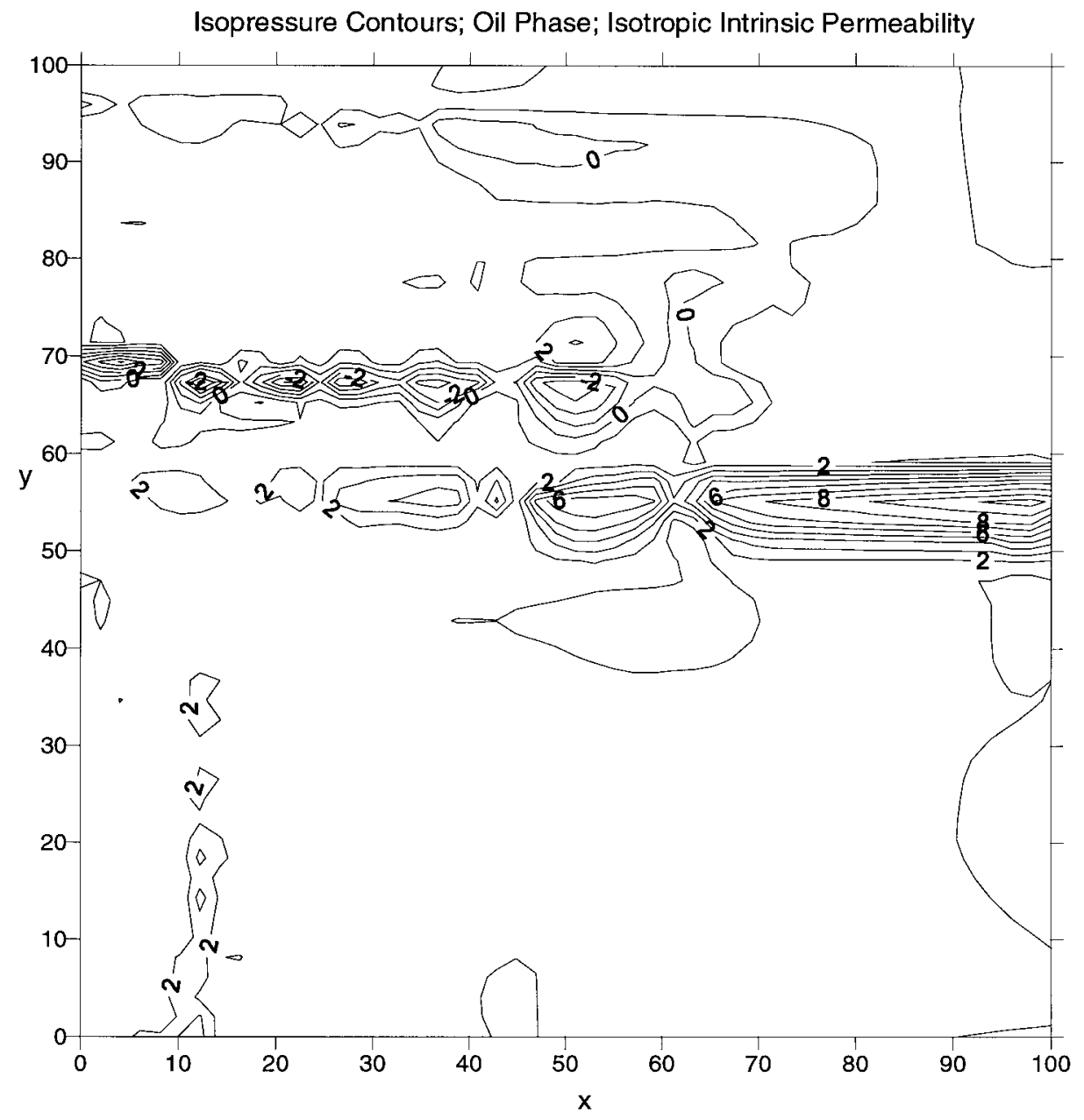

FIG. 11. Oil phase isopressure contours for the isotropic intrinsic permeability field. The pressure is given in units of the entry pressure $p_{e}$.

The flowpaths develop a time dependence induced by the change of the saturation field [41]. The SFP system should be solved subject to an initial saturation profile. The relaxation of the multiphase system in the transient state will be studied in a future publication.

9. Discussion and conclusions. We proposed an SFP method for studying multiphase flow in heterogeneous porous formations that is based on established notions of differential geometry. A numerical algorithm based on discretization of the flowpath equations and the Newton-Raphson method is implemented for twodimensional flow. The method is based on a simple iterative approach: an initial saturation profile is assumed and updated progressively until the saturation relaxes to a stable value. Steady-state conditions are considered in this work, but the SFP method is applicable in the case of transient flow as well. 


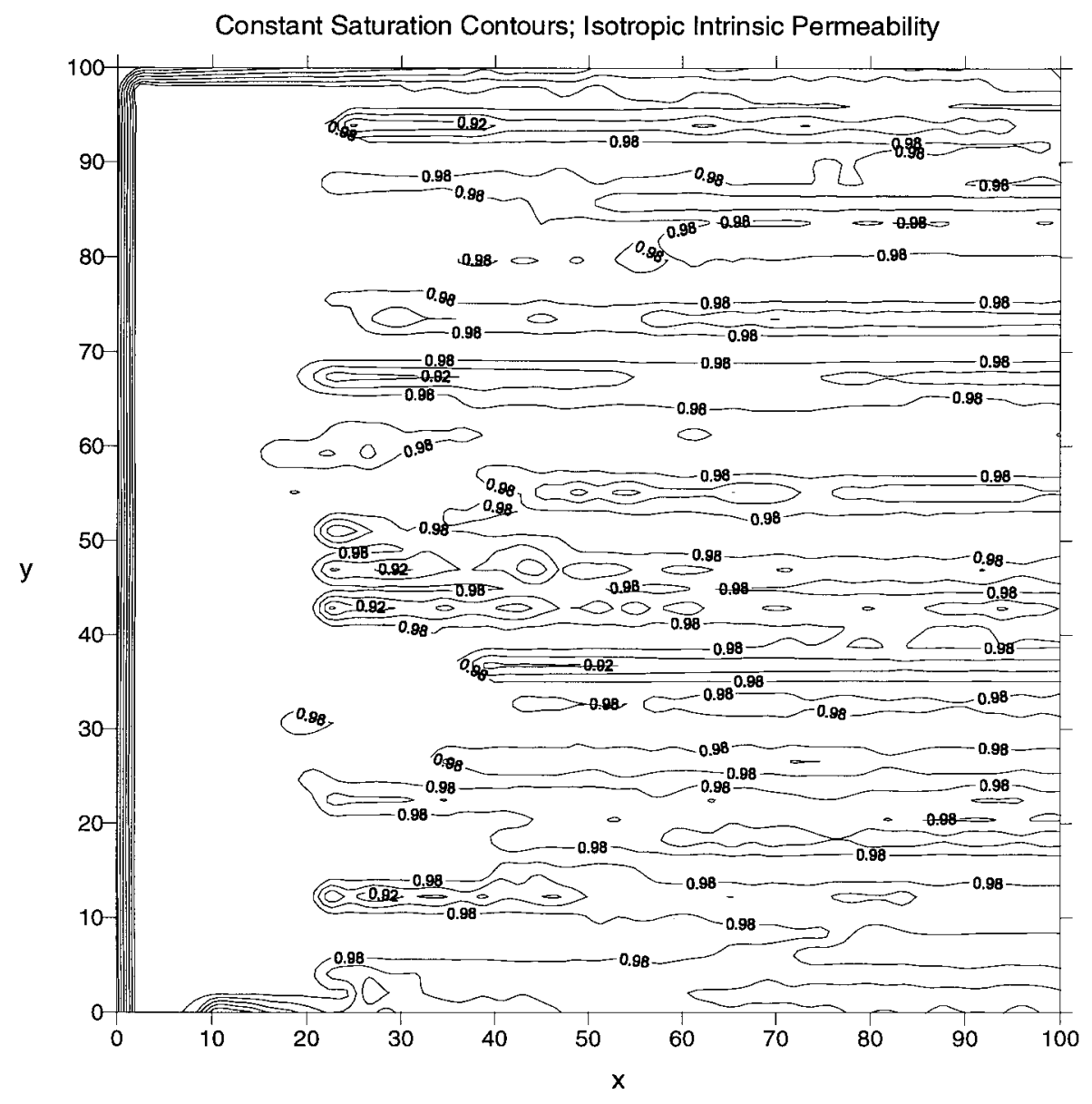

FIG. 12. The reduced water saturation contours for the isotropic intrinsic permeability field.

Other techniques based on the idea of studying multiphase flow along flowpaths (or streamlines) have also been recently introduced in petroleum engineering [23], [24]. These techniques, however, address the solution of the simpler fractional flow model often used in oil recovery. In particular, the fractional flow model is based on the high flow-velocity approximation that neglects the capillary pressure; the multiphase flow equations can then be formulated in terms of a total velocity that is determined from an effective Darcy's law. The SFP method is considerably more general than these techniques. Indeed, the SFP method allows for nonzero values of the capillary pressure. It can also incorporate various sets of constitutive relationships that are of interest in environmental sciences. In principle, hysteretic p-S relations can be accommodated, as well. In this paper an attempt is made to introduce the new method and discuss its main features by means of theoretical results (e.g., sections 3-5) and a numerical example (section 6). To our knowledge, there are no exact analytical results for multiphase flow when the capillary pressure is not ignored. In a future publication, we plan to investigate additional issues related to the convergence, 


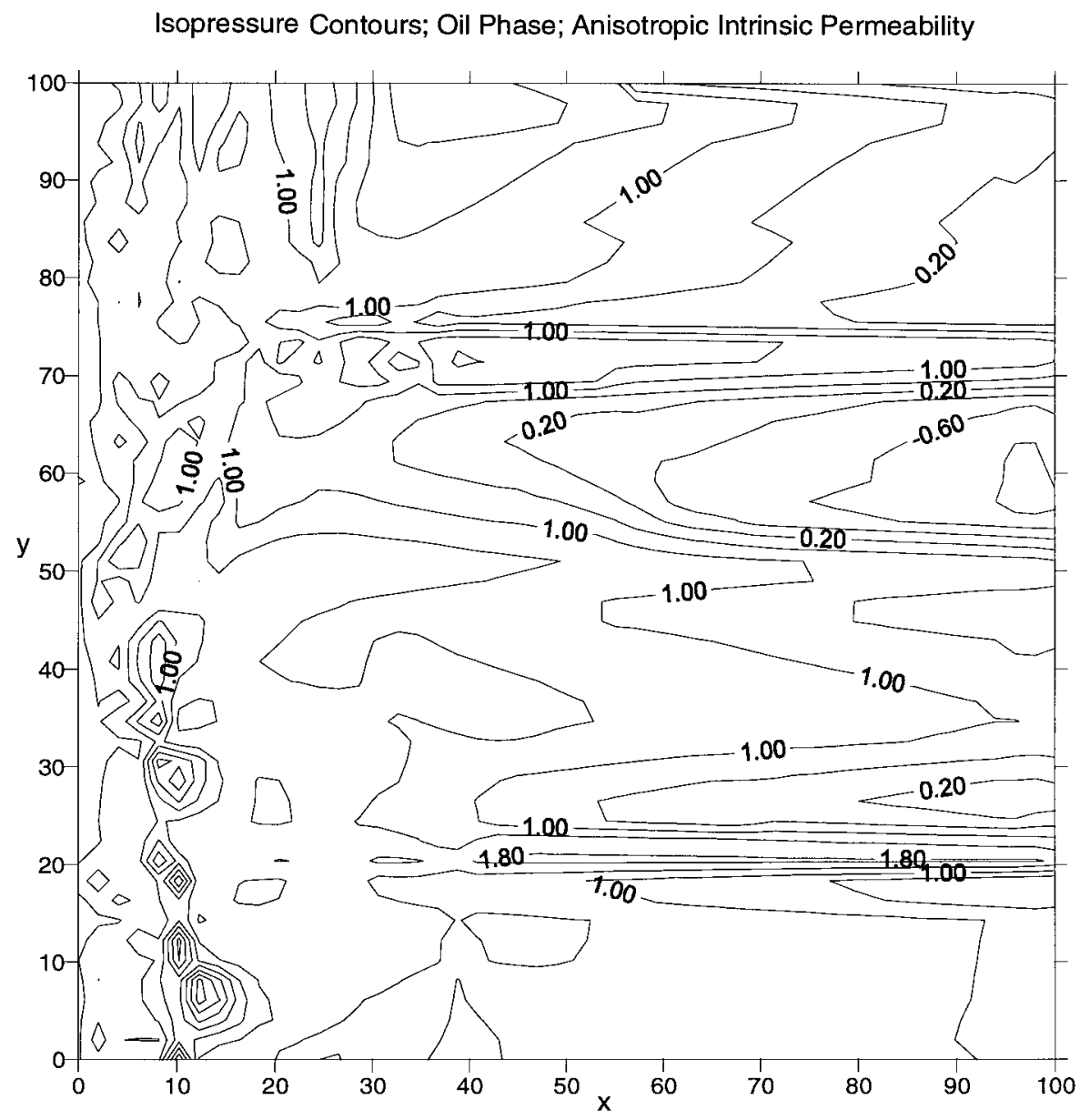

FIG. 13. Oil phase isopressure contours for the anisotropic intrinsic permeability field. The pressure is given in units of the entry pressure $p_{e}$.

accuracy, and stability of the proposed method, as well as to perform computational and experimental comparisons. In particular, more research is required to investigate three-dimensional flow with gravitational effects, the time evolution of the flowpaths, and the parameter basin within which the numerical code converges.

Appendix I. Equations (2) for the pressure gradient can be expressed as

$$
\partial p_{a} / \partial s_{i}=-\zeta_{a} \epsilon_{i, a}-\rho_{a} g \delta_{3 i}
$$

where $\delta_{3 i}$ is the Kronecker delta. The directional derivative of $p_{a}$ with respect to the arc length $\ell_{\alpha}$ in the direction $\mathbf{e}_{a}$ is

$$
\frac{d p_{\alpha}}{d \ell}=\nabla p_{\alpha} \cdot \mathbf{e}_{\alpha}=-\zeta_{\alpha}-\rho_{\alpha} g \epsilon_{3, \alpha}
$$




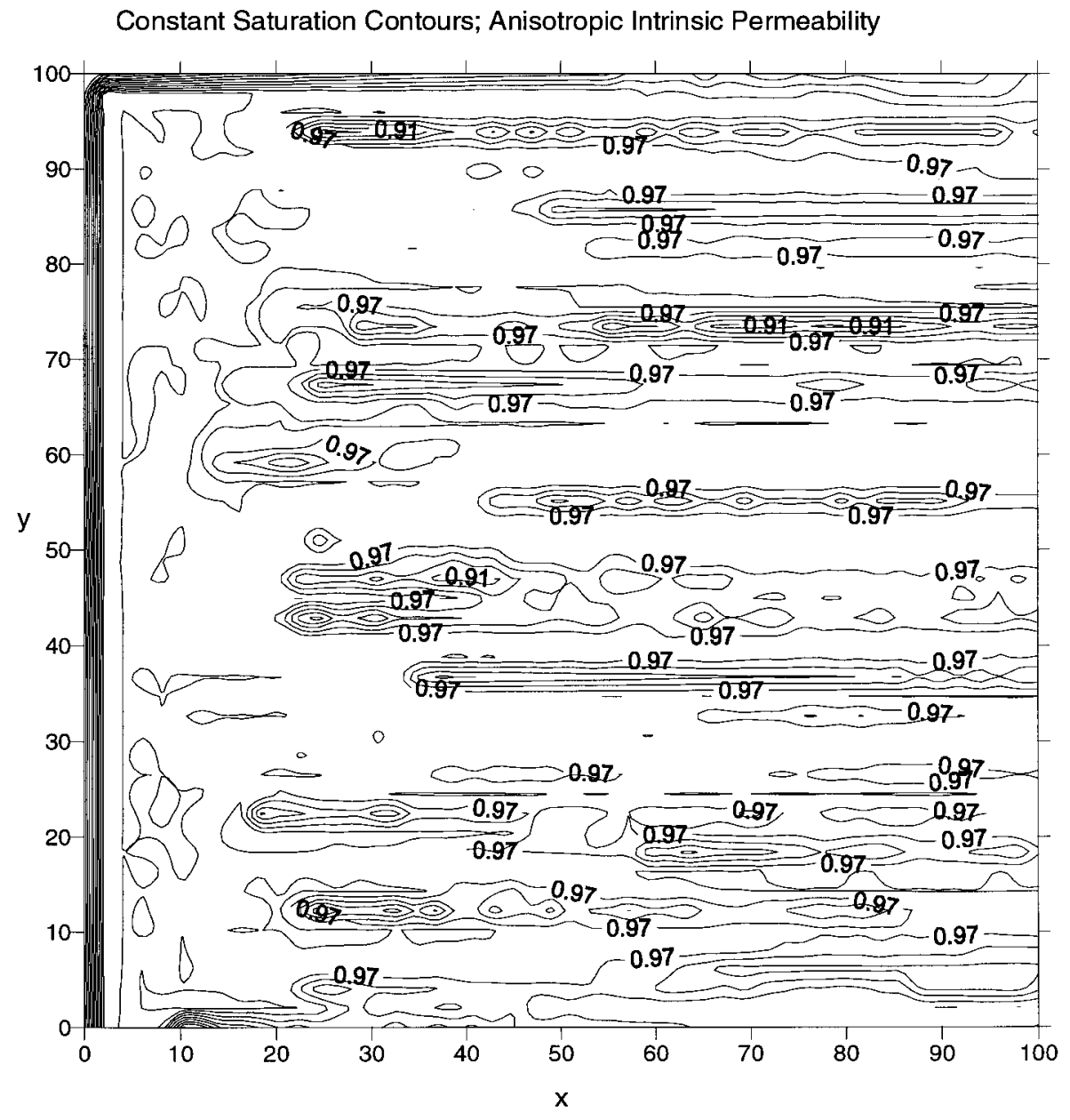

FIG. 14. Reduced water saturation contours iterations for the anisotropic intrinsic permeability field.

Applying the del operator $\nabla$ on both sides of (I.2) we obtain

$$
\frac{d}{d \ell} \nabla p_{a}=-\nabla \zeta_{a}-\rho_{a} g \nabla \epsilon_{3, a}
$$

for all $\alpha$. The above gives the rate of change of the pressure gradient $\nabla p_{\alpha}$ along an $\alpha$-FP. Finally, replacing the gradient on the left-hand side of (I.3) by means of (I.1) we obtain the SFP system of equations (11).

\section{REFERENCES}

[1] M.A. Celia, H. Rajaram, and L.A. Ferrand, A multi-scale computational model for multiphase flow in porous media, Adv. Water Resour., 16 (1993), pp. 81-92. 
[2] J.F. Pankow and J.A. Cherry, Dense Chlorinated Solvents and Other DNAPLs on Groundwater, Waterloo Press, Portland, OR, 1996.

[3] C.T. Miller, G. Christakos, P.T. Imhoff, J.F. McBride, J.A. Pedit, and J.A. TrangenSTEIN, Multiphase flow and transport modelling in heterogeneous porous media: Challenges and approaches, Adv. Water Resour., 21 (1998), pp. 77-120.

[4] A. Bensoussan, J.L. Lions, and G. Papanicolaou, Asymptotic Analysis for Periodic Structures, North-Holland, Amsterdam, The Netherlands, 1978.

[5] R.E. Ewing, Adaptive grid refinements for transient flow problems, in Adaptive Methods for Partial Differential Equations, J.E. Flaherty, P.J. Paslow, M.S. Shephard, and J.D. Vasilakis, eds., SIAM, Philadelphia, PA, 1998, pp. 194-205.

[6] S.M. Hassanizadeh And W.G. Gray, Toward an improved description of the physics of twophase flow, Adv. Water Resour., 16 (1993), pp. 53-67.

[7] R. Peyret and T.D. Taylor, Computational Methods for Fluid Flow, Springer-Verlag, New York, 1983.

[8] D.B. McWhorter and D.K. Sunada, Exact integral solution for two-phase flow, Water Resour. Res., 26 (1990), pp. 399-414.

[9] M.B. Allen, Numerical modelling of multiphase flow in porous media, Adv. Water Resour., 8 (1985), pp. $162-187$.

[10] B.H. Kueper and E.O. Frind, Two-phase flow in heterogeneous porous media, 1. Model development, Water Resour. Res., 27 (1991), pp. 1049-1057.

[11] B.H. KuePer AND E.O. Frind, Two-phase flow in heterogeneous porous media, 2. Model application, Water Resour. Res., 27 (1991), pp. 1059-1070.

[12] G. Papanicolaou, Ed., Random Media, Springer-Verlag, New York, 1987.

[13] G. Papanicolaou, ED., Hydrodynamic Behavior and Interacting Particle Systems, SpringerVerlag, New York, 1987.

[14] H.I. Essaid AND K.M. Hess, Monte-Carlo simulation of multiphase flow incorporating spatial variability of hydraulic properties, Ground Water, 31 (1993), pp. 123-134.

[15] A. Abdin, J.J. KaluarachChi, C-M. Chang, and M.W. Kemblowski, Stochastic analysis of two-phase flow in porous media: II. Comparison between perturbation and Monte-Carlo results, Transport Porous Media, 19 (1995), pp. 261-280.

[16] C.-M. Chang, M.W. Kemblowski, J.J. Kaluarachchi, and A. Abdin, Stochastic analysis of two-phase flow in porous media: I. Spectral/perturbation approach, Stochastic Hydrol. Hydraul., 19 (1995), pp. 233-259.

[17] U. Hornung, ed., Introduction, in Homogenization and Porous Media, Springer-Verlag, New York, 1996.

[18] G. Allaire and A. Mikelic, One-phase Newtonian flow, in Homogenization and Porous Media, U. Hornung, ed., Springer-Verlag, New York, 1996, pp. 45-76.

[19] A. Bourgeat, Two-phase flow, in Homogenization and Porous Media, U. Hornung, ed., Springer-Verlag, New York, 1996, pp. 95-125.

[20] A. Bourgeat, A. Mikelic, and S. Kozlov, Effective equations of two-phase flow in random media, Calc. Var. Partial Differential Equations, 3 (1995), pp. 385-406.

[21] A. Ben-Menahem, Seismic Waves and Sources, Springer-Verlag, New York, 1981.

[22] R. Lenormand, Transport equations for fluid displacements in heterogeneous porous media: The M.H.D. model, in Mathematical Modelling of Flow Through Porous Media, A. Bourgeat, C. Carasso, S. Luckhaus, and A. Mikelic, eds., World Scientific, River Edge, NJ, 1995.

[23] M.J. Blunt, K. Liu, And M.R. Thiele, A generalized method to predict reservoir flow, Petroleum Geosci., 2 (1996), pp. 259-269.

[24] M.R. Thiele, P.R. Batycky, M.J. Blunt, and F.M. OrR, Simulating flow in heterogeneous media using streamtubes and streamlines, SPE Res. Engrg., 10 (1996), pp. 5-12.

[25] L.W. Lake, Enhanced Oil Recovery, Prentice-Hall, Englewood Cliffs, NJ, 1989.

[26] G. Christakos, Random Field Models in Earth Sciences, Academic Press, San Diego, CA, 1992.

[27] G. Dagan, Flow and Transport in Porous Formations, Springer-Verlag, Berlin, 1989.

[28] J. Douglas, Jr., F. Furtado, and F. Pereira, The statistical behavior of instabilities in immiscible displacement subject to fractal geology, in Mathematical Modelling of Flow Through Porous Media, A. Bourgeat, C. Carasso, S. Luckhaus, and A. Mikelic, eds., World Scientific, River Edge, NJ, 1995.

[29] G. Chavent and J. Jaffre, Mathematical Models and Finite Elements for Reservoir Simulation, Elsevier Science Publishers, New York, 1986.

[30] M. Muskat, Physical Principles of Oil Production, McGraw-Hill, New York, 1949. 
[31] F.A.L. Dullien, Porous Media-Fluid Transport and Porous Structure, Academic Press, San Diego, CA, 1992.

[32] D.T. Hristopulos and G. Christakos, A variational calculation of the effective fluid permeability of heterogeneous media, Phys. Rev. E., 55 (1997), pp. 7288-7298.

[33] D.W. Jordan and P. Smith, Nonlinear Ordinary Differential Equations, Oxford University Press, New York, 1987.

[34] J. Bear, Dynamics of Fluids in Porous Media, Dover, New York, 1988.

[35] A.W. Nutbourne and R.R. Martin, Differential Geometry Applied to Curve and Surface Design, Ellis Horwood Ltd., Chichester, West Sussex, England, 1988.

[36] S. Helgason, The Radon Transform, Birkhäuser, Boston, MA, 1980.

[37] G. Christakos, D.T. Hristopulos, And X. Li, Multiphase flow in heterogeneous porous media: A stochastic differential geometry viewpoint, Water Resour. Res., 34 (1998), pp. 93-102.

[38] L.D. Oliver And G. Christakos, Boundary condition sensitivity analysis of the stochastic flow equation, Adv. Water Resour., 19 (1996), pp. 109-120.

[39] M.C. Leverett, Capillary behaviour in porous solids, AIME Trans., 142 (1941), pp. 152-169.

[40] W.H. Press, B.P. Flannery, S.A. Teukolskyy, and W.T. Vetterling, Numerical Recipes, Cambridge University Press, New York, 1986.

[41] G. Christakos and D.T. Hristopulos, Spatiotemporal Environmental Health Modelling: A Tractatus Stochasticus, Kluwer Academic Publishers, Dordrecht, The Netherlands, 1998. 